\title{
Does tumor profile in gastric and gastroesophageal (GE) junction cancer justify off-label use of targeted therapy? - a narrative review
}

\author{
Christopher Del Prete ${ }^{1}$, Arun Muthiah ${ }^{2}$, Khaldoun Almhanna ${ }^{1}$ \\ ${ }^{1}$ Division of Hematology Oncology, Department of Internal Medicine, ${ }^{2}$ Department of Internal Medicine, Warren-Alpert School of Medicine at \\ Brown University/Lifespan Health, Providence, RI, USA \\ Contributions: (I) Conception and design: All authors; (II) Administrative support: All authors; (III) Provision of study materials or patients: C Del \\ Prete, A Muthiah; (IV) Collection and assembly of data: All authors; (V) Data analysis and interpretation: All authors; (VI) Manuscript writing: All \\ authors; (VII) Final approval of manuscript: All authors. \\ Correspondence to: Christopher Del Prete, MD. Division of Hematology Oncology, Department of Internal Medicine, Warren-Alpert School of \\ Medicine at Brown University/Lifespan Health, 593 Eddy Street, Providence, RI 02903, USA. Email: cdelprete@lifepspan.org.
}

\begin{abstract}
Despite significant therapeutic progress, gastric cancer remains among the most deadly forms of cancer encountered in clinical practice, and this remains true even in the context of declining incidence. Outcomes in advanced disease remain poor and therapy is rarely curative in this setting. As our understanding of tumor profile gains sophistication, a growing interest in targeted therapies and moreover the use of tumor profile to inform these therapies has developed in the hopes of altering nearly uniformly poor outcomes. A wide and growing array of molecular targets have been identified in the recent past. Targets of potential clinical interest include human epidermal growth factor receptor-2 (HER2), epidermal growth factor receptor (EGFR), poly(ADP-ribose) polymerase (PARP), mammalian target of rapamycin (mTOR), c-MET, and fibroblast growth factor receptor (FGFR). This advanced molecular understanding has been increasingly used to justify the off-label usage of targeted therapies, though the efficacy of this approach warrants careful consideration. While targeted agents have demonstrated efficacy across a wide range of malignancies, even with molecular profiling data, efficacy is not assured. It will also be demonstrated that even within the same malignancy, what holds true in the metastatic setting does not necessarily apply to the adjuvant or neoadjuvant setting. This review will assess the current evidence for the use of targeted therapies utilizing these biomarkers in the context of gastric and gastroesophageal (GE) junction cancers.
\end{abstract}

Keywords: Gastric cancer (GC); targeted therapy; tyrosine kinase inhibitor; human epidermal growth factor
receptor-2 (HER2); vascular endothelial growth factor (VEGF); epidermal growth factor receptor (EGFR);
poly(ADP-ribose) polymerase (PARP)

Submitted Apr 24, 2020. Accepted for publication Jul 19, 2020.

doi: $10.21037 /$ atm-20-3510

View this article at: http://dx.doi.org/10.21037/atm-20-3510

\section{Introduction}

Despite a decreasing incidence, gastric cancer (GC) remains an important cause of cancer-related death and morbidity. This year in the US alone, approximately 27,600 patients will be diagnosed and 11,010 will die from the disease (1). Importantly, GC is typically diagnosed in incurable stages, which contributes to its rank as the third leading cause of cancer death (2). While incomplete, our understanding of the molecular pathogenesis of GC has evolved rapidly and contributed to a growing array of targeted therapeutic options in late stage disease. Notwithstanding these advances, the story of targeted therapy in GC is marked by repeated failures, and additional treatments are sorely needed. This fact has driven the off-label prescription of 
targeted therapies with or without reference to molecular tumor profile. This article will review the pathobiology of GC, evaluate the evidence for the use of targeted therapies, especially treatment based on biomarkers, and describe the rationale behind off-label use in this deadly disease. We present the following article in accordance with the Narrative Review reporting checklist (available at http:// dx.doi.org/10.21037/atm-20-3510).

\section{Methods}

An English language literature search utilizing the PubMed database for years 2010-2010 was undertaken. Data collection was confined to published research only. Keywords included advanced gastric cancer and molecular/targeted therapy. Prospective and retrospective analyses were included.

\section{Discussion}

\section{Basic pathobiology}

It is crucial to recognize the inherent heterogeneity of GC and to note that no classification system can adequately capture the histological and clinical variety encountered in daily practice. The molecular pathways involved in carcinogenesis can vary widely even within the same histologic type. This may in part explain the failure of many targeted therapies. Nevertheless, an understanding of the basic classification and pathobiology of GC is important toward interpreting or even predicting treatment efficacy. Despite some question as to the prognostic value of such categorization, there is evidence that histologic subtype can indicate molecular tumor profile (3).

Close to $95 \%$ of GCs are adenocarcinomas and within this classification, tumors can be broken down into intestinal or diffuse forms based on a histologic schema first proposed by Lauren in 1965 (4). The molecular mechanisms of this system have since been elucidated and to some extent explain the divergent phenotypes seen in clinical practice. The intestinal type of GC is a well-differentiated tumor with gland structure that resembles normal colon. These cancers have a lesser predilection for local invasion and metastasis and this is thought to be because of preserved expression of E-cadherin, an important mediator of cell-cell adhesion (5). The diffuse type carries a worse prognosis in general and is associated with loss of E-cadherin with a corresponding increased risk of early invasion and metastasis (6). A third category of histologic and genomic classification is the mixed type, which indicates intermediate pathologic findings on the intestinal-diffuse disease spectrum. There is a growing recognition that expression profiling (GEP) shows an incomplete concordance between histologic classification and GEP-based classification, and this may in turn drive future efforts at classification (7).

Importantly, intestinal type histology appears to be associated with $H$. pylori. This ubiquitous organism causes chronic inflammation in a small percentage of individuals, and in an even smaller cohort can initiate the genetic alterations leading to carcinoma. The latter sequence is felt to offer a prototypical model of multistep carcinomatosis. Additional factors at play in inducing the chronic inflammation that can lead to carcinoma include high salt or nitrate consumption and cigarette smoking. $\mathrm{Cag}^{+}$expression on the H. Pylori organism itself is now felt to be a highly important factor in driving carcinomatosis. Unfortunately, the pathogenesis of the diffuse subtype is less well-characterized though is known to involve loss of E-cadherin expression (8).

While more modern classification systems, some based on TP53 expression and microsatellite stability, have evolved, a more important development has been the stratification of patients based on tumor molecular characterization. As discussed further below, though the results of biomarkerbased treatment have been mixed, the hope is that this will allow for a more rational approach to treatment.

\section{Human epidermal growth factor receptor-2 (HER2)}

Despite our growing understanding of the mechanisms of disease in GC, with the exception of immunotherapy, there are only two FDA approved targeted therapies for advanced GC: trastuzumab and ramucirumab (see Figure 1, Table 1). The former targets HER2 and is approved for first-line treatment in advanced HER2-positive GCs (26).

HER2 is an oncogene found on chromosome 17 that that when amplified leads to receptor overexpression. Despite obvious success in targeting HER2 in gastric and breast cancer, the receptor itself is incompletely understood. While it is known to function partly as a ligand independent receptor with kinase activity, the particular ligand that causes direct activation is unknown. The downstream effects of receptor activation are better characterized and include anti-apoptotic, pro-migratory, and proliferation effects (27). The incidence of overexpression varies widely in clinical trials though the best available evidence is from the Trastuzumab for Gastric Cancer (ToGA) trial which found 


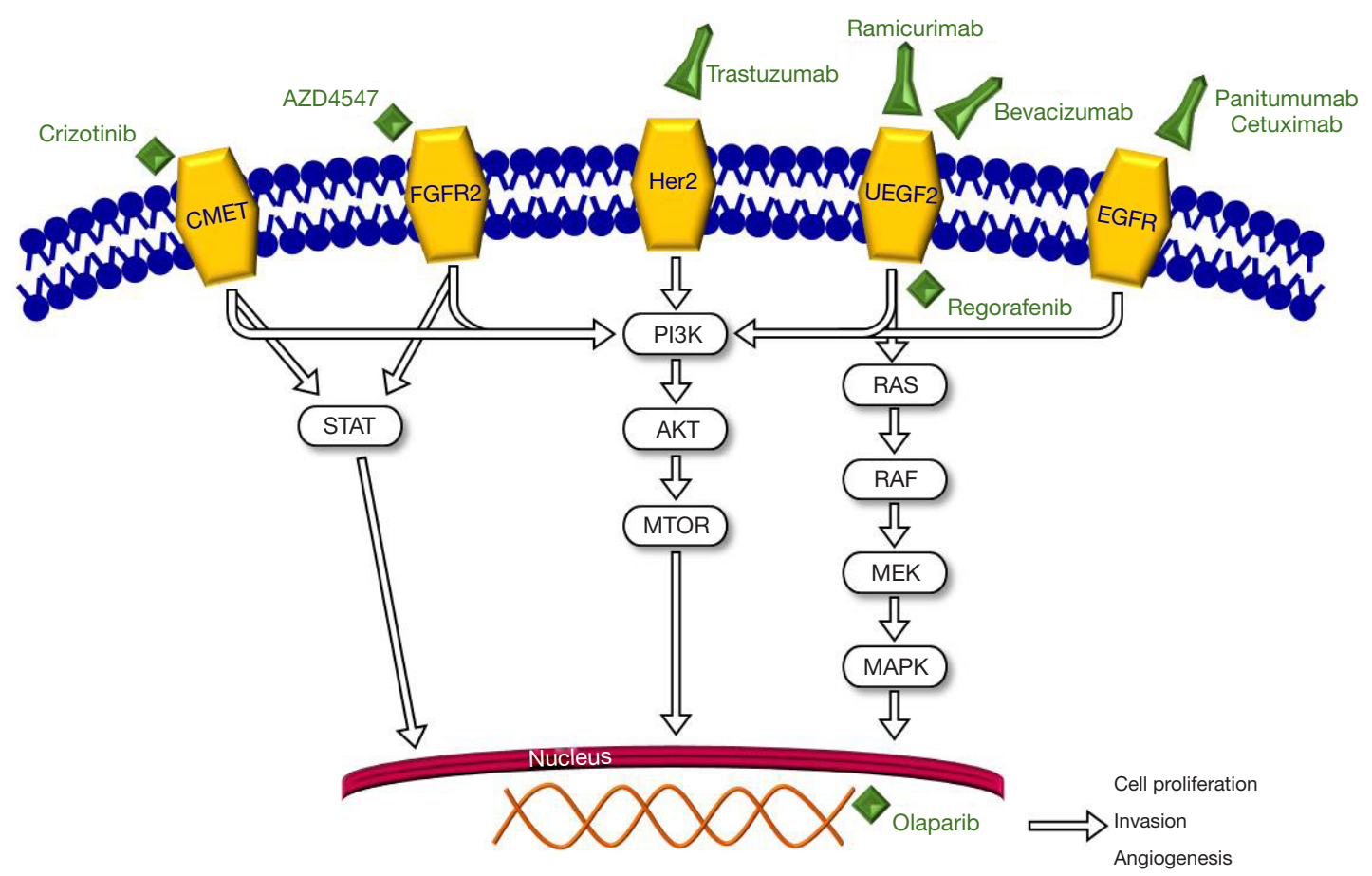

Figure 1 Summary of clinical targets.

overall HER2 expression in 22\% of GCs. Histologic type is related to degree of expression: $32 \%$ of intestinal type tumors have been found to have expression of HER 2 vs. $6 \%$ of diffuse-type (3). Overexpression, a key determinant of response to trastuzumab, is comparatively lower (6-30\%) and seems to be dependent on location of the tumor, with esophagogastric junction (EGJ) cancers more highly expressing HER2 than gastric body cancers (3).

The ToGA trial established front-line trastuzumab as the standard of care in the metastatic setting, showing a median 2.7-month overall survival (OS) advantage for trastuzumab plus chemotherapy $v s$. chemotherapy alone (9). Following approval of trastuzumab, many clinical trials were undertaken to assess response in adjuvant, neoadjuvant and second line metastatic settings. Prior to maturation of this data, off label use in these settings was not uncommon, extrapolated from established benefit in breast cancer. Unfortunately, these trials failed repeatedly to demonstrate any response to other HER2-directed agents. In addition, the role for high-dose trastuzumab was not established after evaluation of this strategy in the IIIb HELOISE trial. Based on the post hoc observation in the ToGA trial that patients with lower trastuzumab serum trough concentrations had shorter OS, the latter compared high $(10 \mathrm{mg} / \mathrm{kg}) v s$. low
$(6 \mathrm{mg} / \mathrm{kg})$ maintenance doses of trastuzumab. There were no significant intergroup differences (10). Because the addition of pertuzumab to trastuzumab improves OS in breast cancer, the authors of the phase III JACOB trial hypothesized that a similar benefit might be seen in HER2positive metastatic gastric or GEJ cancers. Unfortunately, this combination did not improve OS compared with placebo (11).

Despite overall promising data in the metastatic setting, it remains unclear whether the benefit of trastuzumab also applies to adjuvant therapy. Use in this setting would be considered off-label, notwithstanding the recent TOXAG trial. This phase II trial was a single-arm, open label study of adjuvant oxaliplatin, capecitabine and trastuzumab plus radiation. Results were reported in abstract form in February 2018 and were notable only for demonstrating the safety and tolerability of this regimen. Median OS, a secondary outcome, has not yet been reached after 25 months of follow-up (12). It should be noted that this was a small trial, enrolling only 34 patients, and it would seem premature to adopt this as a strategy for the routine care of curatively resected patients. As these data mature, the standard of care may likewise evolve to include trastuzumab or its derivatives for HER2 overexpressors in 
Table 1 Summary of clinical trials of targeted therapies in gastric and gastroesophageal cancer

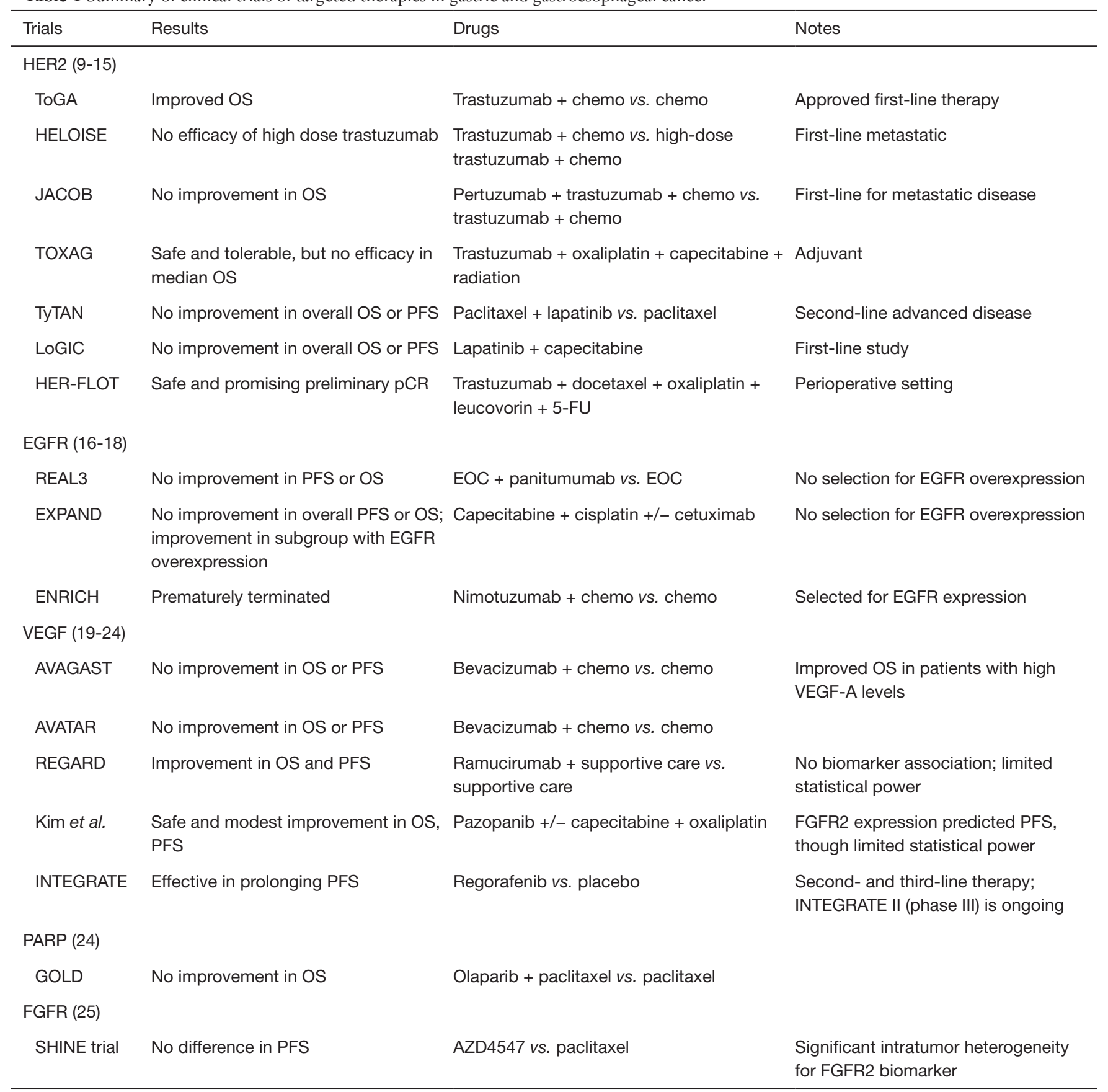

OS, overall survival; PFS, progression-free survival; pCR, pathologic complete response; FGFR, fibroblast growth factor receptor; VEGF, vascular endothelial growth factor; EGFR, epidermal growth factor receptor.

the adjuvant setting.

The phase III TyTAN trial evaluated the combination of paclitaxel with or without lapatinib, a dual HER2 and epidermal growth factor receptor (EGFR) inhibitor, the second line setting (13). There was no statistically significant benefit in OS or progression-free survival (PFS) overall, though a subgroup analysis did demonstrate increased PFS in IHC3+ patients (HR 5.6 vs. 4.2 months, $\mathrm{P}=0.01$ ). This did not translate to an increase in OS however and is of doubtful clinical benefit. 
The phase III LoGIC trial was a first-line evaluation of lapatinib combined with capecitabine and oxaliplatin in advanced GC. Despite an improvement in OS by 1.7 months, statistical significance was not reached, and the improvement in response rate $(53 \%$ vs. $39 \%$ in the placebo arm, $\mathrm{P}=0.0031)$ came at a cost of increased toxicity, with diarrhea a prominent side effect (14). Even more disappointing for any justifiable off-label usage of lapatinib was the lack of a relationship between HER2 status by IHC and OS. EGFR expression status was not evaluated.

Encouragingly, there are several ongoing and/or recently completed clinical trials that seek to answer this question. These include the HER-FLOT trial, RTOG 1010, as well as NCTO01130337 (15). The latter in particular will look at trastuzumab maintenance for 1 year (28). This is likewise a phase II design but will address disease-free survival (DFS) as a primary outcome and may prove to be practice changing. RTOG 1010 compared RT, paclitaxel and carboplatin with or without trastuzumab in patients with GEJ or esophageal adenocarcinoma, stages T1N1-2 or T2-3N0-2. Unfortunately, this trial failed to demonstrate improved DFS when trastuzumab was added to trimodality therapy (29).

One exciting development presented at the American Society of Clinical Oncology (ASCO) 2020 was the presentation of the DESTINY-GASTRIC01 data. This was an open-label, randomized phase II trial of trastuzumab deruxtecan (DS-8201), a conjugate of anti-HER2 antibody and a topoisomerase inhibitor, in advanced GC patients who had received at least two prior therapies. An objective response was noted in $51 \%$ of the trastuzumab deruxtecan group compared to $14 \%$ of the physicians choice group. This translated to a significant OS benefit of $12.5 \mathrm{vs} .8 .4$ months. These results represent a meaningful advancement in the evolution of targeted therapy. Interestingly, the benefit was highest in patients with high HER2 expression, though the results of an exploratory analysis with lower HER2 expressors is as yet unpublished (30).

\section{EGFR}

Also part of the ErB family of tyrosine kinase inhibitors is the EGFR. Estimates of EGFR gene amplification and protein overexpression in GC are $5 \%$ and $27.4 \%$ respectively $(16,31)$. Unfortunately, attempts at targeting EGFR in GC have failed to demonstrate meaningful clinical benefit. There have been two major negative trials including the REAL3 and EXPAND trials $(17,18)$. The former evaluated cetuximab with or without chemotherapy in the first-line advanced GC setting and demonstrate a statistically nonsignificant difference in OS of $10.7 v s$. 9.4 months. The latter added panitumumab, a monoclonal antibody against EGFR, to a chemotherapy backbone and again found no difference in OS or PFS.

Importantly, these trials did not in any way select for EGFR overexpression. It is at least worth mentioning that subgroup analysis of EXPAND did show improvement in PFS and OS in patients with high EGFR overexpression. Given the relatively low expression seen in GC, it was felt with proper patient selection a benefit could exist. Unfortunately, the phase III ENRICH trial, itself based on a phase II trial that did find benefit in subgroup analysis of high EGFR expressors with EGFR inhibition, was terminated due to negative results in a mid-term followup (19). Off-label use of anti-EGFR therapies cannot be justified on the basis on available data.

\section{Vascular endothelial growth factor (VEGF)}

The vascular endothelial growth factor receptor family (VEGFR) and its ligands in the VEGF class are known stimulators of angiogenesis, a process crucial for tumor growth across a broad array of malignancies. There is a known association in GC with both tumor and peripheral VEGF expression and survival (20). Observation of this association has not translated in a meaningful way to clinical benefit.

Bevacizumab targets only VEGF-A and this may in part explain the failure of this agent to improve outcomes in multiple GC trials. The phase III AVAGAST trial added bevacizumab to capecitabine/cisplatin without OS benefit. This study did, in a prospective manner, assess for VEGF-A plasma levels at baseline. While the authors report a trend toward improved OS in patients with high baseline VEGF-A levels $v s$. low, this was not statistically significant (21). Three years later, the AVATAR study demonstrated similar findings, and there was no biomarker assessment made (22). These data taken together do not offer a clinical rationale for offlabel bevacizumab in the first-line setting.

Ramucirumab is an IgG1 monoclonal antibody that functions as a receptor antagonist to VEGFR-2. This is in direct contrast to bevacizumab, which targets only the ligand of VEGFR-2. Ramucirumab is a highly specific and therefore potent inhibitor of VEGFR-2. This compelling mechanism of action has translated to significant clinical benefit in the advanced setting, leading to FDA approval for second line advanced GC. The REGARD trial showed 
a statistically significant improved OS of 1.4 months when ramucirumab was added to best supportive care $v$ s. placebo in patients with refractory advanced GC. The extrapolation of this data to off-label usage is limited by the relatively low number of patients selected for biomarker analysis: in this case, $43 \%$ of patients underwent biomarker testing including VEGFR-2 and HER2 tumor analysis and serum VEGF. Certainly, it is telling that there was no biomarker association with OS/PFS, but statistical power per the authors admission was quite limited (32). Further studies with ramucirumab are underway that may add to this dilemma.

Pazopanib is a tyrosine kinase inhibitor with a wide spectrum of activity that blocks VEGF1-3, fibroblast growth factor receptor (FGFR) 1 and 3, PDGFR and c-kit among others (23). It is commercially available for sarcoma and advanced renal cell carcinoma and has been evaluated for first-line and refractory treatment in combination with chemotherapy. Kim and colleagues performed a phase II trial of pazopanib plus CapeOX for first-line patients with metastatic or advanced GC. Clinical benefit was modest: OS was reported at 10.5 months while PFS was 6.5 months. The regimen was well tolerated (33). Intriguingly, a biomarker analysis was subsequently performed in $81 \%(n=54)$ of patients. FGFR2 expression predicted PFS: among expressors, PFS was $8.5 v s$. 5.6 months among non-expressors (34). Given the limited statistical power, these data need to be validated with larger trials before they are put into clinical practice.

Regorafenib is another multi-kinase inhibitor with a broad spectrum of activity including VEGFR1-3, TIE-2, PDGFR-B, FGFR, as well as RAF, RET and KIT. The phase two INTEGRATE study of advanced esophagogastric cancer in the second- or third-line setting showed encouraging results in advanced GC patients (PFS $v s$. placebo 2.6 and 0.9 months) (24). The INTEGRATE II phase III study is ongoing. The latter will include prognostic and predictive biomarkers as part of secondary endpoint analysis but will not randomize patients according to biomarker status (35). While FDA approved for metastatic colorectal cancer in 2012 and gastrointestinal stromal tumor (GIST) in 2013, there is currently no rationale for off-label use of this in the metastatic or advanced GC setting.

\section{Poly(ADP-ribose) polymerase (PARP) inbibition}

PARP inhibitors prevent repair of single-strand DNA breaks leading to apoptosis (24). Olaparib is FDA approved in recurrent ovarian cancer, metastatic breast cancer, and $B R C A$ mutated metastatic pancreatic cancer. After multiple positive phase II trials, in 2017 the phase III GOLD trial evaluated olaparib plus paclitaxel $v s$. paclitaxel and placebo. OS did not significantly differ. Ataxia-telangiectasia mutated protein (ATM)-negative tumors were hypothesized to be more responsive however secondary analysis revealed no significant different when stratified based on ATM status (36).

\section{Mammalian target of rapamycin (mTOR)}

The mammalian target of rapamycin or mTOR is a PI3K related protein that is intimately involved in cellular proliferation, growth, and metabolism and is known to be deregulated in a wide array of human cancers (37). Everolimus is an mTOR inhibitor with approval across breast, neuroendocrine, and renal cell cancers. Multiple phase III studies have failed to demonstrate a benefit in advanced GC in the second or later line setting (38). Biomarkers have not been adequately assessed in these trials however.

\section{$c-M E T$}

c-MET is a tyrosine kinase receptor that when activated by hepatocyte growth factor (HGF) leads to a variety of downstream effects including the promotion of metastasis, cell growth, and angiogenesis (39). Unfortunately, results of studies targeting this pathway in GC to date have returned negative (40). In the third-line setting, we are awaiting the results of a pilot study of crizotinib using c-MET as a biomarker in advanced GC (25).

\section{FGFR}

FGFR2 is another tyrosine kinase that is amplified in approximately $5-10 \%$ of GCs and is therefore an appealing target for molecular based therapy. The phase II SHINE trial evaluated the FGF2 inhibitor AZD4547 as monotherapy vs. paclitaxel in the advanced setting. Patients were randomized according to FGFR gene amplification or polysomy. PFS was not improved compared to paclitaxel. Interestingly, an exploratory biomarker analysis found a high degree of intratumor heterogeneity for expression of FGFR2 amplification/polysomy suggesting that this is a highly flawed biomarker (41). A recent phase I trial evaluated bemarituzumab, a humanized IGG1 monoclonal antibody against FGFR2B, for activity in advanced GC 
and GEJ adenocarcinoma in the late-line setting. Seventynine patients were enrolled and were stratified according to FGFR2b expression level (high, medium, low, none). Seventeen point nine percent of patients with FGFR2B high level expression demonstrated a partial response (42). We eagerly await an upcoming phase III trial which will combine this agent with chemotherapy.

\section{Conclusions}

A wide array of anti-neoplastic agents targeting the various molecular pathways known to be at play in advanced GC have been assessed. Despite this enormous research effort, a minority have found efficacy. We are thus witnessing the sizable discordance between identified targets and meaningful efficacy. The use the HER2 targeted agent trastuzumab has demonstrated efficacy in the advanced setting, though it seems unlikely that this translates to benefit in the adjuvant or neoadjuvant setting. Whether this hold true for the antibody-drug conjugate trastuzumab deruxtecan is unknown. Targeting of the EGFR and VEGF pathways has resulted in generally disappointing outcomes, though there is hope that initial positive results with pazopanib will be borne out in larger trials. Trials targeting FGFR are ongoing after some encouraging early data, while the same cannot be said of c-MET, mTOR, or PARP directed therapies. While off-label use may appropriately evolve as the above data mature, at present there is little justification for such use.

\section{Acknowledgments}

Special thanks to Kaitlin Zupancic for assisting in the creation of Figure 1.

Funding: None.

\section{Footnote}

Provenance and Peer Review: This article was commissioned by the Guest Editor (Dr. Khaldoun Almhanna) for the series "Gastroesophageal Cancer 2020" published in Annals of Translational Medicine. The article was sent for external peer review organized by the Guest Editor and the editorial office.

Reporting Checklist: The authors have completed the Narrative Review reporting checklist. Available at http:// dx.doi.org/10.21037/atm-20-3510
Conflicts of Interest: All authors have completed the ICMJE uniform disclosure form (available at http://dx.doi. org/10.21037/atm-20-3510). The series "Gastroesophageal Cancer 2020" was commissioned by the editorial office without any funding or sponsorship. KA served as the unpaid Guest Editor of the series and serves as an unpaid editorial board member of Annals of Translational Medicine from Nov 2019 to Oct 2021. The other authors have no other conflicts of interest to declare.

Ethical Statement: The authors are accountable for all aspects of the work in ensuring that questions related to the accuracy or integrity of any part of the work are appropriately investigated and resolved.

Open Access Statement: This is an Open Access article distributed in accordance with the Creative Commons Attribution-NonCommercial-NoDerivs 4.0 International License (CC BY-NC-ND 4.0), which permits the noncommercial replication and distribution of the article with the strict proviso that no changes or edits are made and the original work is properly cited (including links to both the formal publication through the relevant DOI and the license). See: https://creativecommons.org/licenses/by-nc-nd/4.0/.

\section{References}

1. Stomach Cancer - Statistics [Internet]. Cancer.Net. 2020 [cited 22 March 2020]. Available online: https://www. cancer.net/cancer-types/stomach-cancer/statistics

2. Hescheler DA, Plum PS, Zander T, et al. Identification of targeted therapy options for gastric adenocarcinoma by comprehensive analysis of genomic data. Gastric Cancer 2020;23:627-38.

3. Kelly CM, Janjigian Y. The genomics and therapeutics of HER2-positive gastric cancer-from trastuzumab and beyond. J Gastrointest Oncol 2016;7:750-62.

4. Lauren P. The two histological main types of gastric carcinoma: diffuse and so-called intestinal-type carcinoma. an attempt at a histo-clinical classification. Acta Pathol Microbiol Scand 1965;64:31-49.

5. Shah MA, Khanin R, Tang L, et al. Molecular classification of gastric cancer: a new paradigm. Clin Cancer Res 2011;17:2693.

6. DeVita V, Lawrence T, Rosenberg S. Devita, Hellman, and Rosenberg's Cancer. 10th edition. Philadelphia: Wolters Kluwer, 2015:613-4.

7. Tan IB, Ivanova T, Lim KH, et al. Intrinsic subtypes 
of gastric cancer, based on gene expression pattern, predict survival and respond differently to chemotherapy. Gastroenterology 2011;141:476.

8. Wroblewski LE, Peek R, Wilson K, et al. Helicobacter pylori and Gastric Cancer: Factors That Modulate Disease Risk. Clin Microbiol Rev 2010;23:713-39.

9. Bang YJ, Van Cutsem E, Feyereislova A, et al. Trastuzumab in combination with chemotherapy versus chemotherapy alone for treatment of HER2-positive advanced gastric or gastro-oesophageal junction cancer (ToGA): a phase 3, open-label, randomised controlled trial. Lancet 2010;376:687-97.

10. Shah MA, Xu RH, Bang YJ, et al. HELOISE: Phase IIIb Randomized Multicenter Study Comparing Standard-ofCare and Higher-Dose Trastuzumab Regimens Combined With Chemotherapy as First-Line Therapy in Patients With Human Epidermal Growth Factor Receptor 2-Positive Metastatic Gastric or Gastroesophageal Junction Adenocarcinoma. J Clin Oncol 2017;35:2558-67.

11. Tabernero J, Hof PM, Shen L, Ohtsu A, et al. Pertuzumab plus trastuzumab and chemo therapy for HER2-positive metastatic gastric or gastro-oesophageal junction cancer (JACOB): fnal analysis of a double-blind, randomised, placebo-controlled phase 3 study. Lancet Oncol 2018;19:1372-84.

12. Abali H, Yalcin S, Onal C, et al. A study of the combination of oxaliplatin, capecitabine, and trastuzumab and chemoradiotherapy in the adjuvant setting in operated patients with HER2 + gastric or gastroesophageal junction cancer (TOXAG study). J Clin Oncol 2018;36:abstr 26.

13. Satoh T, Xu RH, Chung HC, et al. Lapatinib plus paclitaxel versus paclitaxel alone in the second-line treatment of HER2-amplifed advanced gastric cancer in Asian populations: TyTAN-a randomized, phase III study. J Clin Oncol 2014;32:2039-49.

14. Hecht JR, Bang YJ, Qin SK, et al. Lapatinib in combination with capecitabine plus oxaliplatin in human epidermal growth factor receptor 2-positive advanced or metastatic gastric, esophageal, or gastroesophageal adenocarcinoma: TRIO-013/LOGiC—a randomized phase III trial. J Clin Oncol 2016;34:443-51.

15. Hofheinz R, Hegewisch-Becker S, Thuss-Patience P, et al. HER-FLOT: Trastuzumab in combination with FLOT as perioperative treatment for patients with HER2-positive locally advanced esophagogastric adenocarcinoma: A phase II trial of the AIO Gastric Cancer Study Group. J Clin Oncol 2014;32:abstr 4073.

16. Kim MA, Lee HS, Lee HE, et al. EGFR in gastric carcinomas: prognostic significance of protein overexpression and high gene copy number. Histopathology 2008;52:738-46.

17. Waddell T, Reis-Filho J, Gonzalez-De-Castro D, et al. A Randomised Multicentre Trial of Epirubicin, Oxaliplatin and Capecitabine (EOC) + Panitumumab in advanced Oesophago-Gastric Cancer (REAL3): Updated Results. Ann Oncol 2012;23:ix224.

18. Lordick F, Kang YK, Chung HC, et al. Capecitabine and cisplatin with or without cetuximab for patients with previously untreated advanced gastric cancer (EXPAND): a randomised, open-label phase 3 trial. Lancet Oncol 2013;14:490-9.

19. Kim HJ, Oh S. Novel Systemic Therapies for Advanced Gastric Cancer. J Gastric Cancer 2018;18:1.

20. Mawalla B, Yuan X, Luo X, et al. Treatment outcome of anti-angiogenesis through VEGF-pathway in the management of gastric cancer: a systematic review of phase II and III clinical trials. BMC Res Notes 2018;11:21.

21. Van Cutsem E, de Haas S, Kang YK, et al. Bevacizumab in combination with chemotherapy as first-line therapy in advanced gastric cancer: a biomarker evaluation from the AVAGAST randomized phase III trial. J Clin Oncol 2012;30:2119-27.

22. Shen L, Li J, Xu J, et al. Bevacizumab plus capecitabine and cisplatin in Chinese patients with inoperable locally advanced or metastatic gastric or gastroesophageal junction cancer: randomized, double-blind, phase III study (AVATAR study). Gastric Cancer 2015;18:168-76.

23. Mechanism of Action I VOTRIENT® (pazopanib) tablets [Internet]. Hcp.novartis.com. 2020 [cited 20 March 2020]. Available online: https://www.hcp.novartis.com/products/ votrient/advanced-renal-cell-carcinoma/mechanism-ofaction/

24. Selim JH, Shaheen S, Sheu WC, et al. Targeted and novel therapy in advanced gastric cancer. Exp Hematol Oncol 2019;8:25.

25. A Pilot Study of Crizotinib in Patients With c-MET Positive Gastric Adenocarcinoma as a Third-line Chemotherapy. Clinicaltrials.gov. 2020 [cited 01 April 2020]. Available online: https://clinicaltrials.gov/ct2/show/ NCT02435108

26. Accessdata.fda.gov. 2020 [cited 15 March 2020]. Available online: https://www.accessdata.fda.gov/drugsatfda_docs/ label/2010/103792s5256lbl.pdf

27. Kiyozumi Y, Iwatsuki M, Yamashita K, et al. Update on targeted therapy and immune therapy for gastric cancer, 2018. J Cancer Metastasis Treat 2018;4:31. 
28. Arienti C, Pignatta S, Tesei A. Epidermal Growth Factor Receptor Family and its Role in Gastric Cancer. Front Oncol 2019;9:1308.

29. Safran, H, Winter KA, Wigle DA, et al. Trastuzumab with trimodality treatment for esophageal adenocarcinoma with HER2 overexpression: NRG Oncology/RTOG 1010. J Clin Oncol 2020;38:abstr 4500.

30. Shitara, K, Bang, YJ, Iwasa S, et al. Trastuzumab Deruxtecan in Previously Treated HER2-Positive Gastric Cancer. N Engl J Med 2020;382:2419-30.

31. Cancer Genome Atlas Research Network. Comprehensive molecular characterization of gastric adenocarcinoma. Nature 2014; 513:202-9.

32. Fuchs CS, Tabernero J, Tomasek J, et al. Biomarker analyses in REGARD gastric/GEJ carcinoma patients treated with VEGFR2-targeted antibody ramucirumab. $\mathrm{Br}$ J Cancer 2016;115:974-82.

33. Kim ST, Lee J, Lee SJ, et al. Prospective phase II trial of pazopanib plus CapeOX (capecitabine and oxaliplatin) in previously untreated patients with advanced gastric cancer. Oncotarget 2016;7:24088-96.

34. Kim ST, Ahn S, Lee J, et al. Value of FGFR2 expression for advanced gastric cancer patients receiving pazopanib plus CapeOX (capecitabine and oxaliplatin). J Cancer Res Clin Oncol 2016;142:1231-7.

35. Sjoquist KM, Pavlakis N, Martin AJ, et al. Integrate II: A randomised phase 3 double-blind placebo-controlled study of regorafenib in refractory advanced gastro-oesophageal cancer (AGOC) - An international study organized by the Australasian Gastrointestinal Trials Group (AGITG). J Clin Oncol 2017. doi: 10.1200/JCO.2017.35.15_suppl.

Cite this article as: Del Prete C, Muthiah A, Almhanna K. Does tumor profile in gastric and gastroesophageal (GE) junction cancer justify off-label use of targeted therapy?a narrative review. Ann Transl Med 2020;8(17):1110. doi: 10.21037/atm-20-3510
TPS4136.

36. Bang YJ, Xu RH, Chin K, et al. Olaparib in combination with paclitaxel in patients with advanced gastric cancer who have progressed following first-line therapy (GOLD): a double-blind, randomised, placebo-controlled, phase 3 trial. Lancet Oncol 2017;18:1637-51.

37. Laplante M, Sabatini D. mTOR signaling at a glance. J Cell Sci 2009;122:3589-94.

38. Ohtsu A, Ajani JA, Bai YX, et al. Everolimus for previously treated advanced gastric cancer: results of the randomized, double-blind, phase III GRANITE-1 study. J Clin Oncol 2013;31:3935-43.

39. Inokuchi M. Clinical significance of MET in gastric cancer. World J Gastrointest Oncol 2015;7:317.

40. Catenacci DV, Tebbutt N, Davidenko I, et al. Rilotumumab plus epirubicin, cisplatin, and capecitabine as first-line therapy in advanced MET-positive gastric or gastro-oesophageal junction cancer (RILOMET-1): a randomised, double-blind, placebo-controlled, phase 3 trial. Lancet Oncol 2017;18:1467-82.

41. Van Cutsem E, Bang Y, Mansoor W, et al. A randomized, open-label study of the efficacy and safety of AZD4547 monotherapy versus paclitaxel for the treatment of advanced gastric adenocarcinoma with FGFR2 polysomy or gene amplification. Ann Oncol 2017;28:1316-24.

42. Catenacci DVT, Rasco D, Lee J, et al. Phase I Escalation and Expansion Study of Bemarituzumab (FPA144) in Patients With Advanced Solid Tumors and FGFR2bSelected Gastroesophageal Adenocarcinoma. J Clin Oncol 2020;38:2418-26. 